\title{
High prevalence of multidrug resistant tuberculosis in Djibouti: a retrospective study
}

\author{
Géraldine Boyer-Cazajous ${ }^{1}$, Christophe Martinaud ${ }^{2}$, Céline Déhan ${ }^{3}$, Mohamed Osman Hassan ${ }^{4}$, Yassin \\ Gaas $^{4}$, Marie-Caroline Chenilleau-Vidal ${ }^{1}$, Charles Soler ${ }^{2}$ \\ ${ }^{1}$ Service de Médecine, Hôpital Médico-Chirurgical Bouffard, République de Djibouti \\ ${ }^{2}$ Fédération de biologie clinique, Hôpital d'Instruction des Armées Percy, Clamart, France \\ ${ }^{3}$ Laboratoire de Biologie Médicale, Hôpital Médico-Chirurgical Bouffard, République de Djbouti \\ ${ }^{4}$ Centre National de Traitement de la Tuberculose, Hôpital Paul Faure, Djibouti, République de Djibouti
}

\begin{abstract}
Introduction: The Republic of Djibouti is an African country that exhibits one of the highest incidence rate of tuberculosis in the world. The aim of this study was to evaluate the prevalence of multidrug-resistant tuberculosis among new cases.

Methodology: We studied retrospectively every tuberculosis case diagnosed over a 12-month period in patients hospitalized at the French Military Hospital of Bouffard. During this period, 1,274 samples from 675 patients were tested.

Results: We isolated 266 mycobacteria corresponding to 180 cases of tuberculosis. Thirty-three were fully susceptible and $57 \%$ met the tuberculosis criteria, with $46 \%$ primary resistance. No extensively-drug-resistant tuberculosis was found.

Conclusion: Our results highlight a major concern about the situation in this part of the world.
\end{abstract}

Key words: Mycobacterium tuberculosis; drug-resistant tuberculosis; Africa

J Infect Dev Ctries 2014; 8(2):233-236. doi:10.3855/jidc.3837

(Received 29 May 2013 - Accepted 15 July 2013)

Copyright (C) 2014 Boyer-Cazajous et al. This is an open-access article distributed under the Creative Commons Attribution License, which permits unrestricted use, distribution, and reproduction in any medium, provided the original work is properly cited.

\section{Introduction}

Emerging multidrug-resistant (MDR) Mycobacterium tuberculosis (TB) is one of the major concern of the Health policy [1]. According to the World Health Organization 650,000 people are infected by MDR-TB worldwide and 12 million suffer from tuberculosis. In Africa, $1.9 \%$ of new cases and $9.4 \%$ of diagnosed and treated patients are infected by a MDR strain [2]. The Republic of Djibouti, with a population of approximately 800,000 people, is a country located in the Horn of Africa that exhibits one of the highest incidence rates of tuberculosis in the world close to 300 new cases per 100,000 people per year. In 2011, a single-center study conducted in Djibouti [3] highlighted a very high level of MDR-TB in this country and few cases of extensively drugresistant (XDR) TB. To go further into this debate, we decided to conduct a clinical and bacteriological study whose aim was to evaluate the prevalence of MDR-TB in patients admitted to the French Military Hospital of Bouffard (HMCB), Djibouti.

\section{Study}

We performed a single-center study between April 2010 and April 2011. Every sample sent for examination to HMCB laboratory for tuberculosis testing was included. For each case, we collected data on age, sex, geographical origin, HIV status and, if administered, previous tuberculosis treatment. No follow-up was available. Scientific and ethical approval was obtained from the HMCB ethic committee. Informed consent was obtained from all patients.

Direct smear examination after Ziehl-Nielssen staining was systematically performed. Cultures were done on Lowenstein-Jensen and Coletsos medium during 60 days. Isolates were sent to the Armed Forces Mycobacterium Reference Laboratory of the Military Medical Center Percy (France) for identification and drug susceptibility testing. Identification was based on staining, MPT-64 detection (SD-Bioline-TB AgMPT64, Eurobio Laboratoires, Courtaboeuf, France) and conventional biochemical procedures. For non tuberculosis mycobacterium, the identification was based on conventional biochemical tests, culture 
criteria [4] and molecular hybridation (GenotypeMTBC, Hain lifescience, Nehren, Germany).

Isolate susceptibility was performed in liquid medium (Bactec-M-GIT960, Becton DickinsonFranklin Lakes, USA) for isoniazid, rifampicin, pyrazimamide and streptomycin. When resistance was detected, susceptibility to second-line drugs (aminoglycosid and fluoroquinolones) was performed by molecular hybridation (Genotype kit-MTBRplus, Hain-Lifescience, Nehren, Germany) according to manufacturer's recommendations. Multi-drug resistance was defined by resistance to rifampicin and isoniazid, and extensively drug-resistance when the strain was MDR-TB and resistant to any of the fluoroquinolones and to at least one of three injectable second-line drugs.

\section{Results}

During this 12-month-long study, 1,274 samples from 675 patients were sent to the laboratory. One hundred $(8 \%)$ were positive at smear examination and
$266(21 \%)$ yielded isolates after culture. Finally, 180 cases were diagnosed as tuberculosis infection based on clinical, radiological and biological findings. Sample data, smear examination and culture results are summarized in Table 1. Prior to culture, acid-fast bacilli were found in $46 \%$ of pulmonary tuberculosis with sputum, in $24 \%$ of pulmonary tuberculosis without sputum and in $33 \%$ of ganglionic tuberculosis. Among those 180 patients, 101 benefited of further analysis in France (56\%, representing 257 samples). Unfortunately, the remaining samples could not be analyzed due to transport difficulties.

The average age was 32 (3-83), 57\% of patients were men $(\mathrm{n}=58), 94 \%$ was Djiboutian. Forty six percent $(n=47)$, were hospitalized at the French Military Hospital of Bouffard, 10\% $(\mathrm{n}=10)$ in the Djiboutian National Tuberculosis Center of Paul Faure and $33 \%(\mathrm{n}=34)$ were treated at home. The identification of the sample revealed $89 \%$ of infection by $M$. tuberculosis $(\mathrm{n}=90), 6 \%$ of $M$. canetti and $5 \%$ of non tuberculosis mycobacteria $(M$. chelonae $\mathrm{n}=1$,

Table 1:_Samples received at Bouffard's laboratory: characteristics, culture results and smears examination.

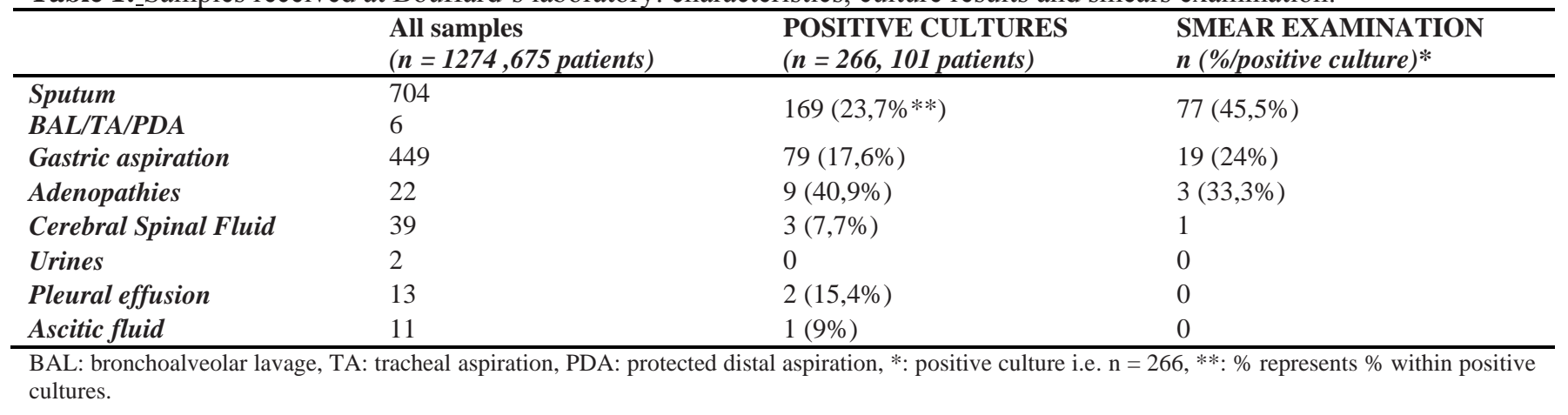

Table 2: Antibiotics susceptibility of isolated Mycobacterium tuberculosis $(\mathrm{n}=88)$

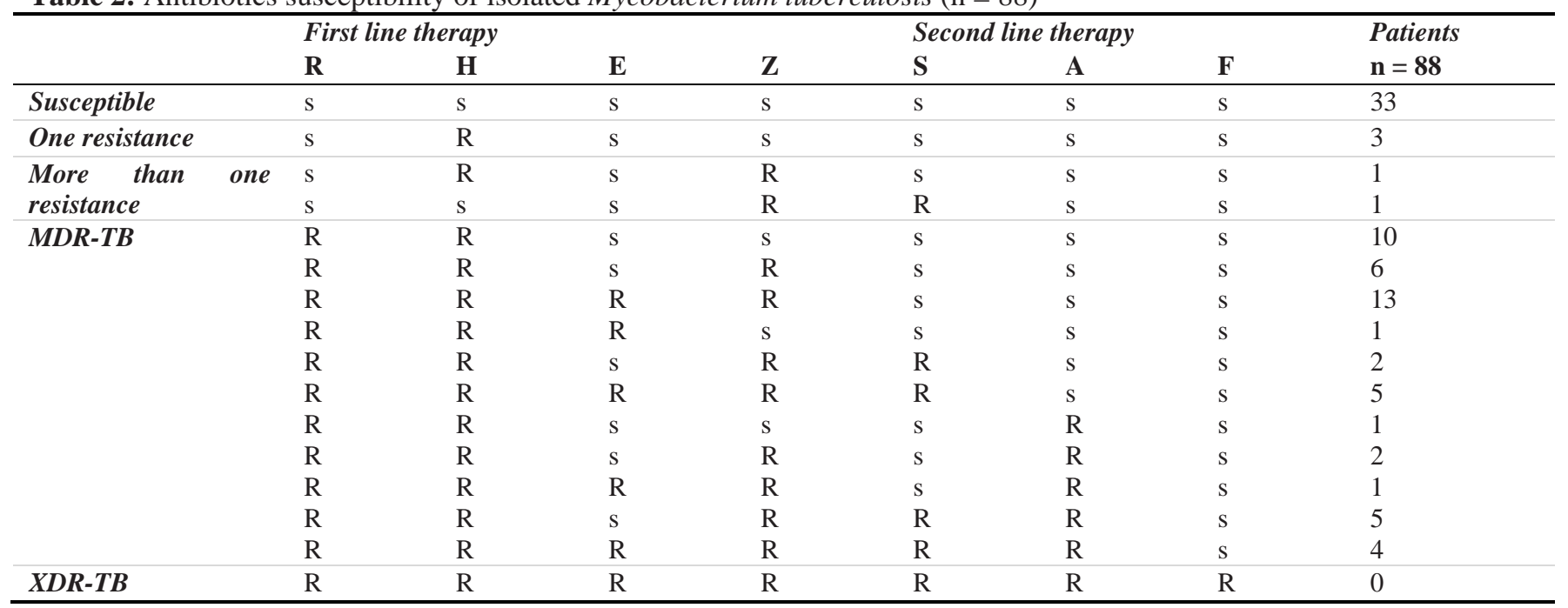

$\mathrm{R}=$ Rifampicin, $\mathrm{H}=$ Isoniazid, $\mathrm{E}=$ Ethambutol, $\mathrm{Z}=$ Pyrazinamide, $\mathrm{S}=$ Streptomycin, $\mathrm{A}=$ Aminosides, $\mathrm{F}=$ fluoroquinolones. In the table, $\mathrm{R}=$ resistant, $\mathrm{s}=$ susceptible. 
M. fortuitum $\mathrm{n}=2, M$. peregrinum $\mathrm{n}=1$ )

A drug susceptibility testing was available for $98 \%$ of patients (88/90) with infection due to $M$. tuberculosis. Susceptibility results are summarized in Table 2. Thirty-three patients (38\%) had an infection with susceptible mycobacteria, five strains harbored a single resistance to isoniazid $(\mathrm{n}=3)$, and 51 isolates $(56,8 \%)$ were MDR-TB. No XDR-TB was identified.

Past medical history, especially anti-tuberculosis therapy was reported in 44 patients, (ten from the National Tuberculosis Center and 34 from the HMCB). Twenty (45\%, four positive for HIV) were infected by a susceptible tuberculosis isolate, and 24 (55\%, four positive for HIV) had a MDR-TB. Among the MDR strains, a previous anti-tuberculosis treatment was reported in 13 patients (54\%), this suggests that $46 \%$ of the MDR TB isolated in our study $(n=11)$ could not be related to a previous exposition to anti-tuberculosis drug and we can hypothesize that these patients had been contaminated by spreading MDR strains

\section{Discussion}

Drug resistance of $M$. tuberculosis is a world health concern. Our study analyzed samples from each patient infected by $M$. tuberculosis during a period of twelve months at the French Military Hospital of Bouffard in Djibouti where both French and Djiboutian people are treated. This population cannot be considered as a representative sample of the entire population but results from this study revealed an emerging health problem. In contrast with the 2011 WHO report [1] our work showed a worrisome high level of MDR-TB responsible for disease in the majority of patients $(57 \%)$. However, the WHO report included only four African countries (Congo, Ethiopia, Nigeria and South Africa) that cannot be compared to Djibouti. The MDR-TB proportion in this study strengthens results found by Olle-Goig et al. [3] (75\% MDR-TB and 11\% XDR-TB) but seems to be more objective since they only tested samples yielded from hospitalized patients, some with a long history of hospitalization, whereas a considerable part of our patients were treated at home. If compared to the first study of tuberculosis drugs susceptibility performed in 2002 [5], the increasing proportion of MDR-TB observed in less than ten years (from 2,4\% in 2002 to $56,7 \%$ in 2011) is really frightening.

However, we are aware of the limitations of our results. First, this is a retrospective study and we could not evaluate the incidence of infection by MDR strains. Secondly, even if $94 \%$ of our patients were
Djiboutian, nearly half had to support the daily hospitalization cost, that cannot be afforded by all patients and has lead to an obvious bias. We have also gathered data from patients moving from the National Tuberculosis Center to the French Military Hospital and this could lead to an overestimation of the MDR rate. In fact, when analyzing samples collected from this center, we found an average of $80 \%$ of MDR-TB infections, as reported by Olle-Goig et al. Thus, out of the 79 remaining patients, $43(54 \%)$ were infected by a MDR strain.

In Djibouti, frequency of tuberculosis is explained by poverty and crowding. Despite free access to health care and DOT (Directly Observed Treatment) strategy, the compliance to treatment is not perfect and facilitates post-treatment resistance. Moreover, the majority of Djiboutians are diagnosed and treated at Paul Faure tuberculosis center and until summer 2012, this center only performed smear microscopy as diagnostic tool without culture and treatment relied exclusively on first-line drugs. This medical process can explain transmission with MDR TB to "undertreated" people without isolation devices. Since summer 2012, the National Tuberculosis Center started to perform rapid test for infection and susceptibility to rifampicin based on polymerase chain reaction (PCR) and culture. A real hope to control the spreading of MDR TB is emerging from this improvement, but some limitations persist since physicians have to select the most critical patients due to the cost and the limited availability of technicians with the required skills [6].

Despite limitations, the results of this study illustrate the work of the only laboratory performing drug susceptibility testing on tuberculosis samples in Djibouti between 2010 and 2011. Rapid changes in diagnosis and treatment of tuberculosis cases are required to limit infection spreading. Moreover, a genotypic study is necessary to understand the emergence and spread of MDR-TB.

\section{Acknowledgements}

We would like to warmly thank technicians from laboratories in Djibouti and in France, especially Rithy Wong who performed most of the tests.

\section{References}

1. WHO (2011) Global Tuberculosis Control. Available http://www.who.int/tb/publications/global_report/2011/gtbr11 _full.pdf. Accessed on 16 January, 2013. 
2. WHO (2008) Epidemiological Fact Sheet on HIV and Aids: core data on epidemiology and response/ Djibouti. WHO globalatlas Available http://apps.who.int/globalatlas/predifi,edReports/EFS/full/EF S2008_DF.pdf. Accessed on 16 January, 2013.

3. Olle-Goig JE, Codina-Grau G, Martin-Casabona N (2011) Resistance to anti-tuberculosis medications in the Horn of Africa. Int J Tuberc Lung Dis 15: 414-416.

4. Levy-Frebault VV, Portaels F (1992) Proposed minimal standards for the genus Mycobacterium and for description of new slowly growing Mycobacterium species. Int J Syst Bacteriol 42: 315-323.

5. Koeck JL, Bernatas JJ, Gerome P, Fabre M, Houmed A, Herve V, Teyssou R (2002) Epidemiology of resistance to antituberculosis drugs in Mycobacterium tuberculosis complex strains isolated from adenopathies in Djibouti. Prospective study carried out in 1999. Med Trop 62: 70-72.

6. Keshavjee S, Farmer PE (2012) Tuberculosis, drug resistance, and the history of modern medicine. N Engl J Med 367: 931936.

\section{Corresponding author}

Christophe Martinaud, MD

Fédération de Biologie Clinique, HIA Percy, 101 avenue Henri

Barbusse, 92140 Clamart, France

Phone: +33141467248

Fax: +33141467237

Email: christophe.martinaud@inserm.fr

Conflict of interests: No conflict of interests is declared. 